\title{
Exploring the bulk of the BL Lacertae object population
}

\section{Gamma-ray properties}

\author{
F. D’Ammando ${ }^{1}$, M. Giroletti ${ }^{1}$, and S. Rainó ${ }^{2,3}$ \\ 1 INAF Istituto di Radioastronomia, via Gobetti 101, 40129 Bologna, Italy \\ e-mail: dammando@ira.inaf.it \\ ${ }^{2}$ Dipartimento Interateneo di Fisica “M. Merlin” dell’Università e del Politecnico di Bari, 70126 Bari, Italy \\ 3 Istituto Nazionale di Fisica Nucleare, Sezione di Bari, 70126 Bari, Italy
}

Received 31 May 2018 / Accepted 1 August 2018

\begin{abstract}
Aims. We are studying an unbiased sample of 42 nearby $(z<0.2)$ BL Lacertae objects with a multi-wavelength approach. The results of Very Long Baseline Interferometry observations were presented in the first paper of this series. In this paper, we study the $\gamma$-ray properties of the sample.

Methods. We analyse data collected by the Fermi-Large Area Telescope (LAT) during its first $8.5 \mathrm{yr}$ of operation in the energy range $0.1-300 \mathrm{GeV}$.

Results. We reveal 23 sources with a test statistic greater than 25 (corresponding to $\sim 4.6-\sigma$ ) out of 42 , with 3 sources not detected in the third LAT active galactic nucleus (AGN) catalogue, and fluxes between $3.5 \times 10^{-10}$ and $7.4 \times 10^{-8} \mathrm{ph} \mathrm{cm}^{-2} \mathrm{~s}^{-1}$. The majority of the sources have hard spectra $(\Gamma \leq 2)$, with only four having values in the range 2.1-2.4. The three newly detected sources have fluxes in the range between $0.54 \times 10^{-9}$ and $1.35 \times 10^{-9} \mathrm{ph} \mathrm{cm}^{-2} \mathrm{~s}^{-1}$ and photon index 1.7-1.9. Among the 23 LAT-detected sources, 19 are included in the third catalogue of hard Fermi-LAT sources, with a spectrum that connects relatively smoothly from $0.1 \mathrm{GeV}$ to $2 \mathrm{TeV}$. LAT-detected BL Lacs are more luminous on parsec scales with respect to non-LAT-detected sources and have larger core dominance according to the unified models.

Conclusions. The LAT-detected BL Lacs seem to be composed of a bulk of "classical" sources dominated by Doppler boosting and characterised by compact and bright radio emission as well as hard $\gamma$-ray spectra. Moreover, we have identified a possible population of low-luminosity BL Lacs not detected by LAT, lacking a VLBI core, and with a small Doppler factor. Furthermore, three LATdetected sources show non-classical properties for $\gamma$-ray emitting BL Lacs (no evidence of relativistic jet, low Doppler factor in radio images, relatively low core dominance) and three other sources, while showing radio emission on parsec scales, are not detected in $\gamma$ rays so far.
\end{abstract}

Key words. BL Lacertae objects: general - gamma rays: galaxies - galaxies: active - galaxies: jets

\section{Introduction}

Blazars are the rarest and yet most extreme class of active galactic nuclei (AGNs), characterised by the presence of a relativistically beamed jet of plasma pointing within a few degrees of our line of sight. On the basis of their optical spectra, they are classified as flat spectrum radio quasars (FSRQs, characterised by strong emission lines) or BL Lacertae objects (BL Lacs, with nearly featureless optical spectra). Besides the difference in optical spectra, the two classes differ in many other observational properties, including luminosity, accretion regime, spectral energy distribution (SED), morphology and kinematics of the radio jet, and evolution over cosmic time (e.g. Ackermann et al. 2015; Ghisellini et al. 2017; Jorstad et al. 2017).

In Liuzzo et al. (2013; hereafter Paper I), a sample of $42 \mathrm{BL}$ Lacs has been selected purely on their optical spectra. The sources were selected from the Roma BZCAT catalogue of known blazars (Massaro et al. 2009) to have a measured redshift $z<0.2$ and to be located within the sky area covered by the Sloan Digital Sky Survey (SDSS, Abazajian et al. 2009). These two criteria allow us to study with high resolution the bulk of the BL Lac population, including the weakest sources, and grant us the availability of good multi-wavelength coverage.

The results of a dual-frequency Very Long Baseline Interferometry (VLBI) survey of the sample were presented in Paper I of this series. Out of the 42 sources in the sample, 27 sources (64\%) were detected on parsec scales. A radio source can remain undetected in a VLBI observation if it is either below the image sensitivity limit defined by the observation setup or if the bulk emission is distributed over scales substantially larger than the restoring beam of the experiment (typically $1-10$ parsec in the case of Liuzzo et al. 2013). Among the sources detected by the VLBI observations, some appeared to be of the "classical" type, that is, bright, compact, with flat spectra and large core dominance (ratio of compact to extended flux). On the other hand, several sources are faint (down to a few mJy and a monochromatic luminosity of $P_{\mathrm{r}} \sim 10^{23.5} \mathrm{~W} \mathrm{~Hz}^{-1}$ ), with moderately steep radio spectra and low core dominance. These properties, in addition to the 15 non-detections, suggest that a significant fraction of BL Lacs are not strongly Doppler boosted.

It is therefore of great importance to study the same population also at high energy. Since the start of operation of the Large Area Telescope (LAT) on board the Fermi Gamma-ray Space Telescope, the number of BL Lacs detected in $\gamma$ rays has rapidly 
Table 1. List of BL Lac sources in our sample.

\begin{tabular}{|c|c|c|c|c|c|c|}
\hline Source name & $\begin{array}{c}\text { RA (J2000) } \\
\text { deg }\end{array}$ & $\begin{array}{c}\text { Dec (J2000) } \\
\text { deg }\end{array}$ & Redshift & 3FGL name & Photon index & $\begin{array}{c}\text { Flux }(E>0.1 \mathrm{GeV}) \\
\left(10^{-9} \mathrm{ph} \mathrm{cm}^{-2} \mathrm{~s}^{-1}\right)\end{array}$ \\
\hline $\mathrm{J} 0751+1730$ & 117.8545 & 17.5142 & 0.185 & - & & \\
\hline $\mathrm{J} 0751+2913$ & 117.7899 & 29.2265 & 0.194 & - & & \\
\hline J0753+2921 & 118.3525 & 29.3589 & 0.161 & - & & \\
\hline J0754+3910 & 118.6545 & 39.1799 & 0.096 & - & & \\
\hline J0809+3455 & 122.4120 & 34.9270 & 0.083 & 3FGL J0809.6+3456 & $1.67 \pm 0.13$ & $1.56 \pm 0.60$ \\
\hline $\mathrm{J} 0809+5218$ & 122.4548 & 52.3163 & 0.138 & 3FGL J0809.8+5218 & $1.88 \pm 0.02$ & $34.90 \pm 1.83$ \\
\hline $\mathrm{J} 0810+4911$ & 122.7275 & 49.1844 & 0.115 & - & & \\
\hline J0847+1133 & 131.8039 & 11.5639 & 0.199 & 3FGL J0847.1+1134 & $1.74 \pm 0.12$ & $2.86 \pm 0.95$ \\
\hline $\mathrm{J} 0850+3455$ & 132.6508 & 34.9230 & 0.145 & 3FGL J0850.2+3500 & $1.92 \pm 0.20$ & $2.09 \pm 1.10$ \\
\hline J0903+4055 & 135.8113 & 40.9333 & 0.188 & - & & \\
\hline $\mathrm{J} 0916+5238$ & 139.2164 & 52.6412 & 0.19 & - & & \\
\hline $\mathrm{J} 0930+4950$ & 142.6565 & 49.8404 & 0.187 & 3FGL J0930.0+4951 & $1.45 \pm 0.21$ & $0.56 \pm 0.40$ \\
\hline $\mathrm{J} 1012+3932$ & 153.2432 & 39.5442 & 0.171 & - & & \\
\hline $\mathrm{J} 1022+5124$ & 155.5526 & 51.4001 & 0.142 & - & & \\
\hline $\mathrm{J} 1053+4929$ & 163.4339 & 49.4989 & 0.14 & 3FGL J1053.7+4929 & $1.80 \pm 0.10$ & $3.63 \pm 0.93$ \\
\hline $\mathrm{J} 1058+5628$ & 164.6572 & 56.4698 & 0.143 & 3FGL J1058.6+5627 & $1.95 \pm 0.03$ & $32.40 \pm 1.62$ \\
\hline $\mathrm{J} 1120+4212$ & 170.2003 & 42.2034 & 0.124 & 3FGL J1120.8+4212 & $1.62 \pm 0.06$ & $5.04 \pm 0.79$ \\
\hline $\mathrm{J} 1136+6737$ & 174.1253 & 67.6179 & 0.136 & 3FGL J1136.6+6736 & $1.72 \pm 0.08$ & $3.21 \pm 0.73$ \\
\hline $\mathrm{J} 1145-0340$ & 176.3963 & -3.6671 & 0.167 & - & & \\
\hline $\mathrm{J} 1156+4238$ & 179.1940 & 42.6354 & 0.172 & - & & \\
\hline J1201-0007 & 180.2758 & -0.1171 & 0.165 & - & & \\
\hline $\mathrm{J} 1201-0011$ & 180.4319 & -0.1872 & 0.164 & - & & \\
\hline $\mathrm{J} 1215+0732$ & 183.7958 & 7.5346 & 0.136 & - & & \\
\hline $\mathrm{J} 1217+3007$ & 184.4670 & 30.1168 & 0.13 & 3FGL J1217.8+3007 & $1.97 \pm 0.02$ & $57.70 \pm 2.75$ \\
\hline $\mathrm{J} 1221+3010$ & 185.3414 & 30.1770 & 0.182 & 3FGL J1221.3+3010 & $1.97 \pm 0.02$ & $15.70 \pm 1.67$ \\
\hline $\mathrm{J} 1221+2813$ & 185.3820 & 28.2329 & 0.102 & 3FGL J1221.4+2814 & $2.10 \pm 0.03$ & $52.50 \pm 2.65$ \\
\hline $\mathrm{J} 1221+0821$ & 185.3836 & 8.3623 & 0.132 & - & & \\
\hline $\mathrm{J} 1231+6414$ & 187.8808 & 64.2384 & 0.163 & 3FGL J1231.5+6414 & $1.94 \pm 0.20$ & $2.11 \pm 1.18$ \\
\hline $\mathrm{J} 1253+0326$ & 193.4458 & 3.4417 & 0.066 & 3FGL J1253.7+0327 & $1.84 \pm 0.10$ & $5.30 \pm 1.45$ \\
\hline $\mathrm{J} 1257+2412$ & 194.3830 & 24.2111 & 0.141 & - & & \\
\hline $\mathrm{J} 1341+3959$ & 205.2713 & 39.9959 & 0.172 & 3FGL J1341.0+3955 & $2.54 \pm 0.18$ & $7.42 \pm 2.33$ \\
\hline $\mathrm{J} 1419+5423$ & 214.9442 & 54.3874 & 0.153 & 3FGL J1419.9+5425 & $2.31 \pm 0.06$ & $19.6 \pm 2.20$ \\
\hline $\mathrm{J} 1427+5409$ & 216.8761 & 54.1566 & 0.106 & - & & \\
\hline $\mathrm{J} 1427+3908$ & 216.9413 & 39.1423 & 0.165 & - & & \\
\hline $\mathrm{J} 1428+4240$ & 217.1361 & 42.6724 & 0.129 & 3FGL J1428.5+4240 & $1.58 \pm 0.09$ & $2.62 \pm 0.64$ \\
\hline $\mathrm{J} 1436+5639$ & 219.2408 & 56.6569 & 0.15 & 3FGL J1436.8+5639 & $1.99 \pm 0.13$ & $4.59 \pm 1.46$ \\
\hline $\mathrm{J} 1442+1200$ & 220.7012 & 12.0112 & 0.163 & 3FGL J1442.8+1200 & $1.80 \pm 0.12$ & $3.57 \pm 1.14$ \\
\hline $\mathrm{J} 1510+3335$ & 227.6716 & 33.5846 & 0.114 & - & & \\
\hline $\mathrm{J} 1516+2918$ & 229.1733 & 29.3026 & 0.13 & - & & \\
\hline $\mathrm{J} 1534+3715$ & 233.6967 & 37.2652 & 0.143 & 3FGL J1535.0+3721 & $2.11 \pm 0.12$ & $5.36 \pm 1.47$ \\
\hline $\mathrm{J} 1604+3345$ & 241.1938 & 33.7561 & 0.177 & - & & \\
\hline $\mathrm{J} 1647+2909$ & 251.8619 & 29.1639 & 0.132 & - & & \\
\hline
\end{tabular}

Notes. Sources are sorted by increasing RA. Column 1: the source name; Cols. 2 and 3: Right ascension and declination in J2000; Col. 4: redshift taken from BZCAT; Col. 5: 3FGL name; Col 6: 3FGL photon index; Col 7: 3FGL integrated flux.

increased, reaching 632 in the third LAT AGN Catalog (3LAC, Ackermann et al. 2015): BL Lacs are now the most abundant population of $\gamma$-ray sources in the sky. Among the sources of our sample, 20/42 are listed in the 3LAC but it is natural to expect that, in the course of the ongoing all-sky survey executed by the LAT, more and more of the sources could be detected in $\gamma$ rays. We list our sources in Table 1.

The main goal of the present paper is therefore to characterise the high-energy properties of the sample with a larger dataset and an improved LAT-data analysis. We present a dedicated analysis of $8.5 \mathrm{yr}$ of Fermi data for all the sources in the sample, used to characterise the $\gamma$-ray detection rate, the $\gamma$-ray flux, and the photon index. The paper is organised as follows. We present LAT data in Sect. 2 and the results in Sect. 3. We then discuss the results in Sect. 4, and compare them to the 3LAC, the $\gamma$-ray data above $10 \mathrm{GeV}$ reported in the third catalogue of Hard Fermi-LAT sources (3FHL; Ajello et al. 2017) and the radio properties of our sample. Finally, we draw our conclusions in Sect. 5.

Throughout the paper, we use a $\Lambda$ cold dark matter cosmology with $h=0.68, \Omega_{\mathrm{m}}=0.31$, and $\Omega_{\Lambda}=0.69$ (Ade et al. 2016). The $\gamma$-ray photon index $\Gamma$ is defined such that $\mathrm{d} N / \mathrm{d} E \propto E^{-\Gamma}$. The quoted uncertainties are given at the $1 \sigma$ level, unless otherwise stated. 


\section{Fermi-LAT data}

The LAT, the primary instrument onboard the Fermi $\gamma$-ray observatory, is an electron-positron pair conversion telescope sensitive to $\gamma$-rays of energies from $20 \mathrm{MeV}$ to $>300 \mathrm{GeV}$. The LAT consists of a high-resolution silicon microstrip tracker, a CsI hodoscopic electromagnetic calorimeter, and an anticoincidence detector for the identification of charged-particle background. The full description of the instrument and its performance can be found in Atwood et al. (2009). The large field of view ( 2.4 sr) allows the LAT to observe the full sky in survey mode every $3 \mathrm{~h}$. The LAT point spread function (PSF) strongly depends on both the energy and the conversion point in the tracker, and less so on the incidence angle. For $1 \mathrm{GeV}$ normal-incidence conversions in the upper section of the tracker, the PSF $68 \%$ containment radius is $0.8^{\circ 1}$.

The Fermi-LAT data presented here have been obtained in a time period of 102 months from 2008 August 5 to 2017 January 31 in the $0.1-300 \mathrm{GeV}$ energy range. During this time, the LAT instrument operated almost entirely in survey mode. The Pass 8 data (Atwood et al. 2013), based on a complete and improved revision of the entire LAT event-level analysis, were used. The analysis was performed with the ScienceTools software package version v10r0p5. Only events belonging to the "Source" class (evclass=128, evtype=3) were used. We selected only events within a maximum zenith angle of $90^{\circ}$ to reduce contamination from the Earth limb $\gamma$ rays, which are produced by cosmic rays interacting with the upper atmosphere. The spectral analysis was performed with the instrument response functions P8R2_SOURCE_V6 using a binned maximum-likelihood method implemented in the Science tool gtlike. Isotropic ("iso_source_v06.txt") and Galactic diffuse emission ("gll_iem_v06.fit") components were used to model the background (Acero et al. 2016) ${ }^{2}$. The normalization of both components was allowed to vary freely during the spectral fitting.

We analysed a region of interest of $30^{\circ}$ radius centred at the location of each target. We used the coordinates of the $\gamma$-ray sources associated to the radio source as reported in the third Fermi-LAT catalogue of sources (3FGL; Acero et al. 2015). All BL Lacs of our sample included in the 3FGL have power-law spectra. If the source is not included in the 3FGL we used the radio coordinates of the source. We evaluated the significance of the $\gamma$-ray signal from the source by means of a maximumlikelihood test statistic (TS) defined as TS $=2 \times\left(\log L_{1}-\right.$ $\log L_{0}$ ), where $L$ is the likelihood of the data given the model with $\left(L_{1}\right)$ or without $\left(L_{0}\right)$ a point source at the position of the target (e.g. Mattox et al. 1996). The source model used in gtlike includes all the point sources from the 3FGL catalogue that fall within $40^{\circ}$ of the target. The spectra of these sources were parametrized by a power-law, a log-parabola, or a super exponential cut-off, according to the model description in the 3FGL catalogue. We also included new candidates within $10^{\circ}$ of our target from the preliminary LAT 8-year point source List $(\mathrm{FL} 8 \mathrm{Y})^{3}$.

For the sources under analysis, we modelled the data with a power-law function with both the normalization factor and photon index left free in the likelihood fit. A first maximum likeli-

\footnotetext{
1 https://www.slac.stanford.edu/exp/glast/groups/ canda/lat_Performance.htm

2 http://fermi.gsfc.nasa.gov/ssc/data/access/lat/ BackgroundModels.html

3 https://fermi.gsfc.nasa.gov/ssc/data/access/lat/ $\mathrm{fl} \mathrm{y} /$
}

hood analysis was performed over the whole period to remove the sources with TS $<25$ from the model. A second maximum likelihood analysis was performed on the updated source model. In the fitting procedure, the normalization factors and the spectral parameters of the sources lying within $10^{\circ}$ of the target were left as free parameters. For the sources located between $10^{\circ}$ and $40^{\circ}$ from our target, we kept the normalization and the spectral shape parameters fixed to the values from the catalogue.

For the new detected sources, we ran a point source localization using the gtfindsrc tool over the photons with $E>1 \mathrm{GeV}$ extracted during the whole period. We also tried to parameterize the spectrum of these sources with a log parabola model, but the fit with a log parabola did not provide a statistically significant improvement with respect to a power-law. These three sources are included in the FL8Y list.

\section{Results of the Fermi-LAT data analysis}

Here, the $\gamma$-ray detection rate, fluxes, and photon indexes of the sources in our sample are reported. We detected 23 out of 42 sources with $\mathrm{TS} \geq 25$ (corresponding to a $\sim 4.6-\sigma$ confidence level for two degrees of freedom). The results of our analysis for the LAT-detected sources are summarised in Table 2. For the sources not detected in our $\gamma$-ray analysis, a 2- $\sigma$ upper limit of the integrated flux (assuming a photon index $\Gamma=2$ ) is reported in Table 3. For two sources the likelihood analysis results in a TS between 10 and 25: J0754+3910 (TS = 12) and J0810+4911 (TS = 11). Adding more data, these sources could be detected by Fermi-LAT in the future.

We confirm the detection of all the sources previously reported in the 3FGL. In addition, three $\gamma$-ray sources not in the 3FGL and associated with BL Lacs in our sample have been revealed, raising the overall detection rate for the sample from $48 \%$ to $55 \%$. We ran a localization of the three new detected sources, and the results are reported in Table 4 . This further analysis confirms a strict spatial association between the $\gamma$-ray source and the radio counterpart associated with the BL Lac. Two out of three sources $(\mathrm{J} 0916+5238$ and $\mathrm{J} 1215+0732)$ are included in the Brazil ICRANet Gamma-ray Blazar (1BIGB) catalogue (Arsioli \& Chang 2017).

In Fig. 1, we show the distribution of the integral fluxes in the $0.1-300 \mathrm{GeV}$ energy range (left panel), the photon indexes (middle panel), and the $\gamma$-ray luminosity (right panel) for the sources in our sample including 2- $\sigma$ upper limits of the flux and corresponding luminosity for non-LAT-detected sources. The integral fluxes of the LAT-detected sources span the range between $3.5 \times 10^{-10}$ and $7.4 \times 10^{-8} \mathrm{ph} \mathrm{cm}^{-2} \mathrm{~s}^{-1}$, with a peak at $\sim 4 \times 10^{-9} \mathrm{ph} \mathrm{cm}^{-2} \mathrm{~s}^{-1}$. The photon index distribution has a peak at around $\Gamma=1.7-2.0$, with only four sources having values in the range 2.1-2.4. The average photon index of the LATdetected sources in our sample is $\langle\Gamma\rangle=1.87 \pm 0.06$. The three new detected sources have fluxes below $1.4 \times 10^{-8} \mathrm{ph} \mathrm{cm}^{-2} \mathrm{~s}^{-1}$, luminosity below $2.5 \times 10^{44} \mathrm{erg} \mathrm{s}^{-1}$, and photon index 1.7-1.9.

For the sources not detected by LAT the 2- $\sigma$ upper limit of the flux ranges between $0.2 \times 10^{-9}$ and $2.7 \times 10^{-9} \mathrm{ph} \mathrm{cm}^{-2} \mathrm{~s}^{-1}$. The lack of detection of these sources in $\gamma$ rays is in agreement with the LAT sensitivity limit $^{4}$ estimated for $10 \mathrm{yr}$ of observations assuming a photon index $\Gamma=2$, that is, $1-2 \times$ $10^{-9} \mathrm{ph} \mathrm{cm}^{-2} \mathrm{~s}^{-1}$.

All sources with luminosity $>5 \times 10^{44} \mathrm{erg} \mathrm{s}^{-1}$ have been detected in $\gamma$ rays by LAT. On the other hand, no sources have

\footnotetext{
4 http://www.slac.stanford.edu/exp/glast/groups/canda/ lat_Performance.htm
} 
Table 2. New analysis results for the LAT-detected sources.

\begin{tabular}{cccc}
\hline \hline Source name & TS & $\begin{array}{c}\text { Flux } \\
\left(10^{-9} \mathrm{~cm}^{-2} \mathrm{~s}^{-1}\right)\end{array}$ & Photon index \\
\hline $\mathrm{J} 0809+3455$ & 83 & $1.96 \pm 0.51$ & $1.90 \pm 0.09$ \\
$\mathrm{~J} 0809+5218$ & 6513 & $28.26 \pm 1.02$ & $1.85 \pm 0.02$ \\
$\mathrm{~J} 0847+1133$ & 242 & $4.11 \pm 0.82$ & $1.89 \pm 0.08$ \\
$\mathrm{~J} 0850+3455$ & 109 & $2.63 \pm 0.57$ & $1.93 \pm 0.08$ \\
$\mathrm{~J} 0916+5238$ & 34 & $0.54 \pm 0.19$ & $1.67 \pm 0.11$ \\
$\mathrm{~J} 0930+4950$ & 28 & $0.35 \pm 0.17$ & $1.50 \pm 0.07$ \\
$\mathrm{~J} 1053+4929$ & 216 & $2.71 \pm 0.41$ & $1.82 \pm 0.06$ \\
$\mathrm{~J} 1058+5628$ & 5001 & $24.45 \pm 0.91$ & $1.92 \pm 0.02$ \\
$\mathrm{~J} 1120+4212$ & 1246 & $4.45 \pm 0.39$ & $1.57 \pm 0.03$ \\
$\mathrm{~J} 1136+6737$ & 356 & $2.20 \pm 0.19$ & $1.68 \pm 0.03$ \\
$\mathrm{~J} 1215+0732$ & 57 & $1.35 \pm 0.44$ & $1.81 \pm 0.11$ \\
$\mathrm{~J} 1217+3007$ & 15910 & $74.12 \pm 1.29$ & $1.93 \pm 0.01$ \\
$\mathrm{~J} 1221+3010$ & 3127 & $16.53 \pm 0.98$ & $1.70 \pm 0.02$ \\
$\mathrm{~J} 1221+2813$ & 4812 & $43.94 \pm 1.54$ & $2.12 \pm 0.02$ \\
$\mathrm{~J} 1231+6414$ & 87 & $1.92 \pm 0.47$ & $1.94 \pm 0.09$ \\
$\mathrm{~J} 1253+0326$ & 370 & $8.23 \pm 1.23$ & $2.02 \pm 0.06$ \\
$\mathrm{~J} 1341+3959$ & 62 & $2.59 \pm 0.56$ & $2.16 \pm 0.12$ \\
$\mathrm{~J} 1419+5423$ & 1982 & $31.54 \pm 1.30$ & $2.41 \pm 0.03$ \\
$\mathrm{~J} 1428+4240$ & 512 & $2.02 \pm 0.29$ & $1.53 \pm 0.05$ \\
$\mathrm{~J} 1436+5639$ & 439 & $4.80 \pm 0.66$ & $1.89 \pm 0.06$ \\
$\mathrm{~J} 1442+1200$ & 280 & $3.40 \pm 0.53$ & $1.79 \pm 0.06$ \\
$\mathrm{~J} 1534+3715$ & 220 & $7.17 \pm 0.87$ & $2.18 \pm 0.06$ \\
$\mathrm{~J} 1647+2909$ & 29 & $0.81 \pm 0.27$ & $1.86 \pm 0.11$ \\
\hline
\end{tabular}

Notes. Column 1: the source name; Col. 2: TS; Col. 3: flux in the 0.1$300 \mathrm{GeV}$ energy range; Col. 4: $\gamma$-ray photon index.

been detected in $\gamma$ rays below $8 \times 10^{43} \mathrm{erg} \mathrm{s}^{-1}$ so far, suggesting that a significant portion of the bulk of the BL Lac object population has not been probed yet with Fermi-LAT. There is no clear distinction between the distribution of the LAT-detected and non-LAT-detected sources in the range of luminosity $1-5 \times 10^{44} \mathrm{erg} \mathrm{s}^{-1}$.

The LAT-detected and non-LAT-detected sources are equally distributed in redshift $(0.066<z<0.199$ for the former, $0.096<z<0.187$ for the latter), suggesting that the redshift has no impact on the $\gamma$-ray detection in our sample. A Kolmogorov-Smirnov (K-S) test (Press et al. 1992) does not show a significant difference between the redshift distributions of LAT-detected and non-LAT-detected BL Lacs in our sample.

If we consider the distribution of flux density at $1.4 \mathrm{GHz}$ obtained by NRAO VLA Sky Survey (NVSS), it is evident that the radio flux densities of LAT-detected sources is usually higher than those of the non-LAT detected sources in our sample (Fig. 2). However there are 12 LAT-detected BL Lacs with flux density $<85 \mathrm{mJy}$ (i.e. the flux limit of the VLBA Imaging and Polarimetry Survey, VIPS; Helmboldt et al. 2007), showing that our sample is representative of a population of LAT-detected BL Lacs for which pc-scale radio properties were unexplored in detail up to our VLBA observations.

\section{Discussion}

The project described in this work is aimed to improve our knowledge of the BL Lac population, through VLBI and FermiLAT observations of a sample of BL Lacs, independently of their $\gamma$-ray properties and with multi-wavelength information available on all targets.
Table 3. Analysis results for the non-LAT-detected sources.

\begin{tabular}{cc}
\hline \hline Source name & $\begin{array}{c}\text { Upper limit integrated flux } \\
\left(10^{-9} \mathrm{ph} \mathrm{cm}^{-2} \mathrm{~s}^{-1}\right)\end{array}$ \\
\hline $\mathrm{J} 0751+1730$ & 0.23 \\
$\mathrm{~J} 0751+2913$ & 0.80 \\
$\mathrm{~J} 0753+2921$ & 0.24 \\
$\mathrm{~J} 0754+3910$ & 1.34 \\
$\mathrm{~J} 0810+4911$ & 1.14 \\
$\mathrm{~J} 0903+4055$ & 1.16 \\
$\mathrm{~J} 1012+3932$ & 0.38 \\
$\mathrm{~J} 1022+5124$ & 0.99 \\
$\mathrm{~J} 1145-0340$ & 1.94 \\
$\mathrm{~J} 1156+4238$ & 0.71 \\
$\mathrm{~J} 1201-0007$ & 2.35 \\
$\mathrm{~J} 1201-0011$ & 2.34 \\
$\mathrm{~J} 1221+0821$ & 2.72 \\
$\mathrm{~J} 1257+2412$ & 0.51 \\
$\mathrm{~J} 1427+5409$ & 2.68 \\
$\mathrm{~J} 1427+3908$ & 0.65 \\
$\mathrm{~J} 1510+3335$ & 1.08 \\
$\mathrm{~J} 1516+2918$ & 0.38 \\
$\mathrm{~J} 1604+3345$ & 2.34 \\
\hline
\end{tabular}

Notes. Column 1: the source name; Col. 2: 2- $\sigma$ upper limit of the integrated flux in the $0.1-300 \mathrm{GeV}$ energy range.

\subsection{Comparison with $3 L A C$}

For the 20 sources already reported in the 3LAC, we show in Fig. 3 the plot of the flux and photon index obtained in this work with $8.5 \mathrm{yr}$ of Pass 8 LAT data with respect to the values reported in the 3FGL for $4 \mathrm{yr}$ of Pass 7 reprocessed LAT data. In Fig. 4, we show the ratio of the $\gamma$-ray fluxes obtained in $8.5 \mathrm{yr}$ and $4 \mathrm{yr}$ and the photon index variation in the two periods of LAT observations. The fluxes are in general in agreement, with a mean value of the ratio between the value over $8.5 \mathrm{yr}$ and that reported in the 3FGL of $1.01 \pm 0.28$. The most prominent outliers are: J1419+5423, whose flux has increased by $60 \%$, and $\mathrm{J} 1341+3959$, whose flux decreased by a factor of three when considering $8.5 \mathrm{yr}$ of LAT data. This is in agreement with the moderate variability usually observed in the LAT energy range for BL Lacs (e.g. Ackermann et al. 2015).

In the right panel of Fig. 3, we plot the 8.5-yr photon index versus the values reported in the 3FGL. The photon indexes are generally consistent, having $\langle\Delta \Gamma\rangle=\left\langle\Gamma_{8.5-\mathrm{yr}}-\Gamma_{3 \mathrm{FGL}}\right\rangle=0.08 \pm$ 0.16 . Only for $\mathrm{J} 1341+3959$ there is a hint of change of the photon index, from $\Gamma_{3 \mathrm{FGL}}=2.54 \pm 0.18$ to $\Gamma_{8.5-\mathrm{yr}}=2.16 \pm 0.12$.

In general, variations in $\gamma$-ray flux and in photon index are not correlated. Neither softer-when-brighter nor harder-whenbrighter trends are found, as shown by the comparison between the $\gamma$-ray flux ratio and the photon index variation presented in Fig. 4, with the exception of J1341+3959 that shows a softerwhen-brighter trend.

In Fig. 5, we compare the $\gamma$-ray photon index and luminosity obtained over $8.5 \mathrm{yr}$ for the 23 LAT-detected BL Lacs in our sample with values in the 3LAC for the other BL Lacs with known redshift, separating sources with $z<0.2$ and $z>0.2$. The average apparent $\gamma$-ray isotropic luminosity in the $0.1-300 \mathrm{GeV}$ energy range of the LAT-detected sources in our sample ranges between $6 \times 10^{43}$ and $6 \times 10^{45} \mathrm{erg} \mathrm{s}^{-1}$, which are typical luminosities for BL Lacs and within the range of 
Table 4. Localization of the new $\gamma$-ray sources.

\begin{tabular}{ccccc}
\hline \hline Source name & $\begin{array}{c}\text { RA } \\
\text { deg }\end{array}$ & $\begin{array}{c}\text { Dec } \\
\text { deg }\end{array}$ & $\begin{array}{c}\text { 95\% error circle radius } \\
\text { deg }\end{array}$ & $\begin{array}{c}\text { Angular separation } \\
\text { deg }\end{array}$ \\
\hline J0916+5238 & 139.189 & 52.647 & 0.038 & 0.018 \\
$\mathrm{~J} 1215+0732$ & 183.773 & 7.519 & 0.039 & 0.027 \\
$\mathrm{~J} 1647+2909$ & 251.861 & 29.170 & 0.042 & 0.006 \\
\hline
\end{tabular}

Notes. Column 1: the source name; Cols. 2 and 3: right ascension and declination of the $\gamma$-ray source; Col. 4: LAT 95\% error circle radius; Col. 5: angular separation between the coordinates of the $\gamma$-ray source and those of the radio counterpart.
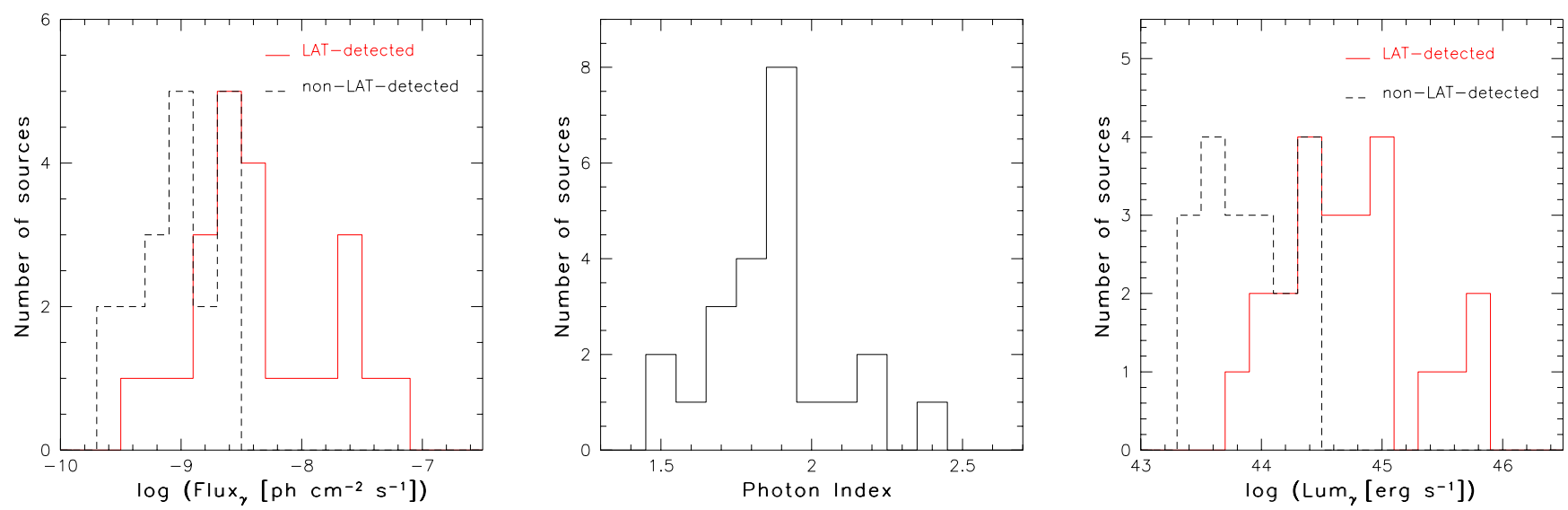

Fig. 1. Histogram of the distribution of various quantities obtained by the LAT analysis for the sources in our sample. Short dash line indicates non-LAT-detected sources (TS < 25), for which 2- $\sigma$ upper limits of the flux are considered; red solid line indicates LAT-detected sources (TS $\geq 25$ ). Left panel: integral fluxes in the $0.1-300 \mathrm{GeV}$ energy range; middle: $\gamma$-ray photon index for the 23 LAT-detected sources; right: $\gamma$-ray luminosity in the $0.1-300 \mathrm{GeV}$ energy range.

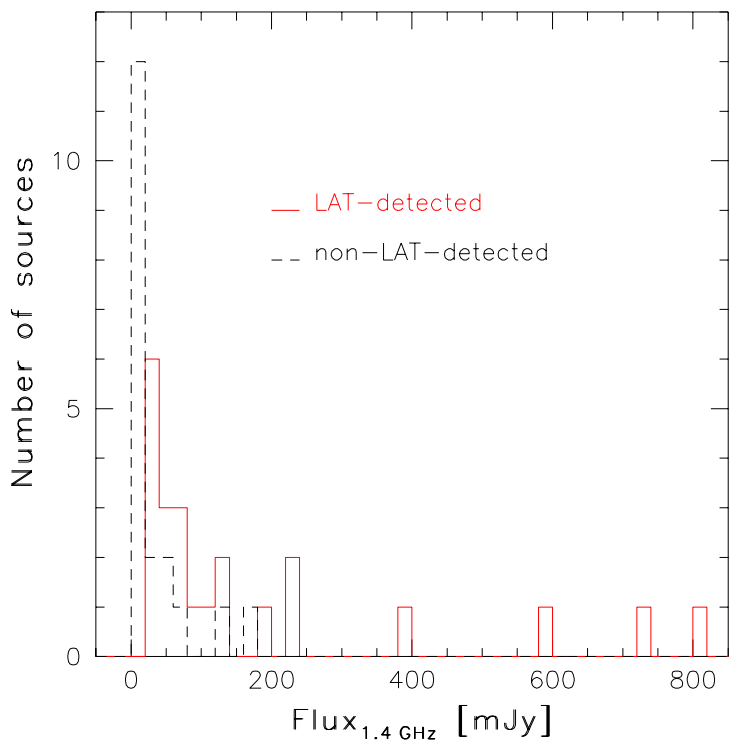

Fig. 2. Histogram of the NVSS flux at $1.4 \mathrm{GHz}$ of the sources in our sample. Short dash line indicates non-LAT-detected sources $(\mathrm{TS}<25)$, red solid line indicates LAT-detected sources $(\mathrm{TS} \geq 25)$.

values observed for the other BL Lacs with $z<0.2$ in the $3 \mathrm{LAC}\left(6 \times 10^{42}-5 \times 10^{46} \mathrm{erg} \mathrm{s}^{-1}\right.$; Ackermann et al. 2015). In the same way, the average photon index of the LAT-detected sources ranges between 1.5 and 2.4, in agreement with what is observed for BL Lacs in the 3LAC. In particular, the average photon index of our sample, $\left\langle\Gamma_{8.5-\mathrm{yr}}\right\rangle=1.87 \pm 0.06$, is compatible with the average photon index reported in the $3 \mathrm{LAC}$ for the other BL
Lacs with $z<0.2,\left\langle\Gamma_{3 \mathrm{LAC}, \mathrm{z}<0.2}\right\rangle=1.95 \pm 0.11$. This suggests that the $\gamma$-ray spectra of the LAT-detected BL Lacs in our sample are similar to the spectra of the other BL Lacs in the 3LAC, in particular at $z<0.2$. Further observations at other wavelengths are needed to investigate differences and similarities between the properties of this sample and those of the other BL Lacs detected by LAT.

Based on the frequency of the synchrotron peak $\left(v_{\text {peak }}^{\text {syn }}\right), \mathrm{BL}$ Lacs can be divided into low-synchrotron-peaked (LSP; $v_{\text {peak }}^{\text {syn }}<$ $10^{14} \mathrm{~Hz}$ ), intermediate-synchrotron-peaked (ISP; $10^{14}<v_{\text {peak }}^{\text {syn }}<$ $10^{15} \mathrm{~Hz}$ ), and high-synchrotron-peaked (HSP; $v_{\text {peak }}^{\text {syn }}>10^{15} \mathrm{~Hz}$ ) BL Lacs (Abdo et al. 2010). Based on the $v_{\text {peak }}^{\text {syn }}$ values reported in the 3LAC, among the LAT-detected sources in our sample 16 are HSP, 3 ISP, and 1 LSP BL Lac. Two out of the three new LAT-detected sources are classified as HSP BL Lac in the second Wide-field Infrared Survey Explorer (WISE) HSP catalogue (2WHSP; Chang et al. 2017). Among the 19 BL Lacs not detected by LAT, 11 are classified as HSP or HSP candidates in the 2WHSP; therefore in our sample 30 of 42 sources (71\%) are classified as HSP or candidate HSP BL Lacs. Lowsynchrotron-peaked sources are usually the brightest BL Lacs detected by LAT. The rising part and high-energy peak of the SED of HSP BL Lacs lies within the LAT energy range, favouring their detection by LAT, as shown by the large number of HSP BL Lacs in the 3LAC. Among the 30 HSP BL Lacs in our sample, 19 sources $(63 \%)$ are detected in $\gamma$ rays, while only 4 out of the 12 (33\%) ISP/LSP BL Lacs have been detected by FermiLAT so far.

An anti-correlation between the synchrotron peak frequency and the LAT photon index measured over $8.5 \mathrm{yr}$ is observed for the LAT-detected BL Lacs in our sample (Fig. 6), as 

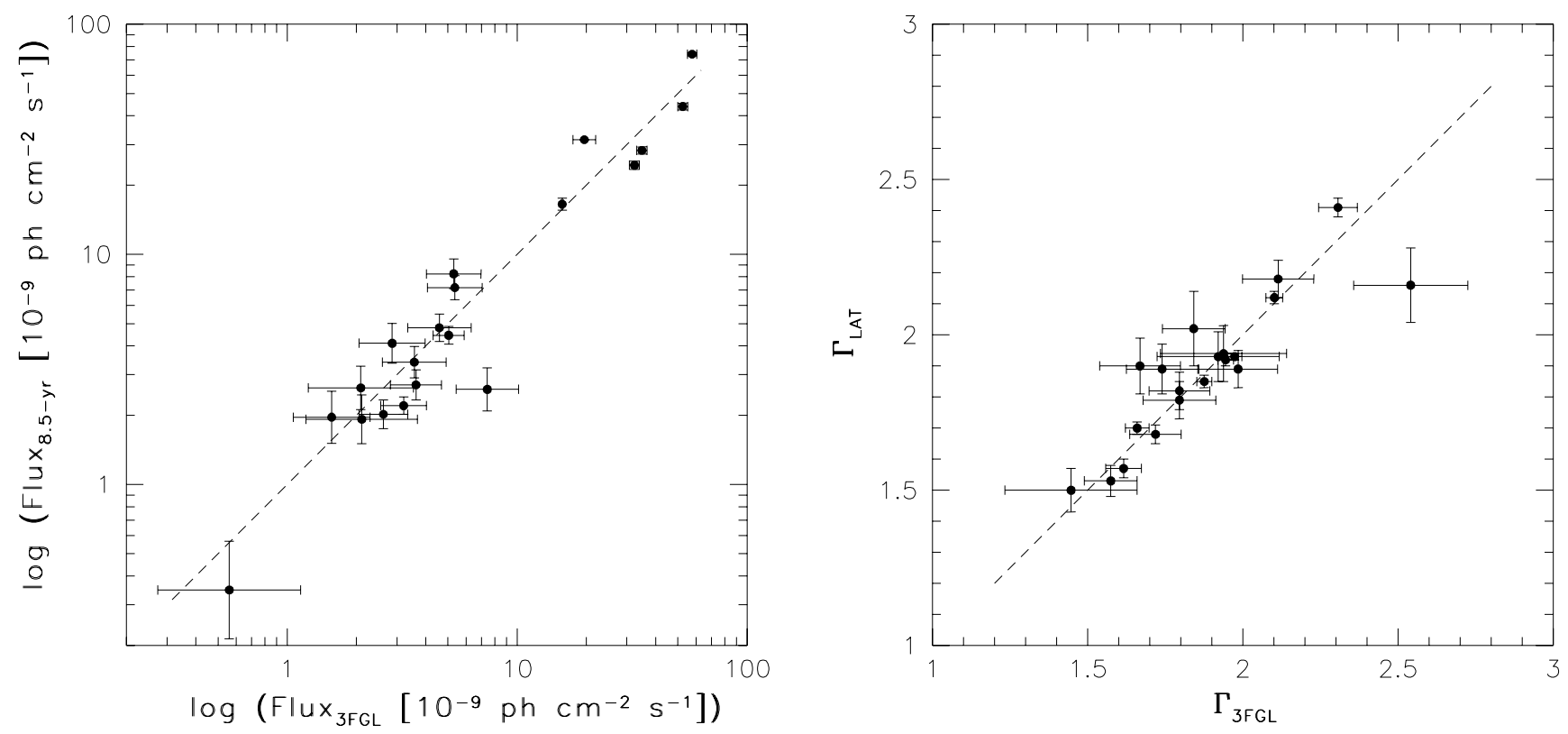

Fig. 3. $\gamma$-ray flux (left panel) and photon index (right panel) obtained in this work vs. the values reported in the 3FGL. A one-to-one reference short dashed line is plotted.

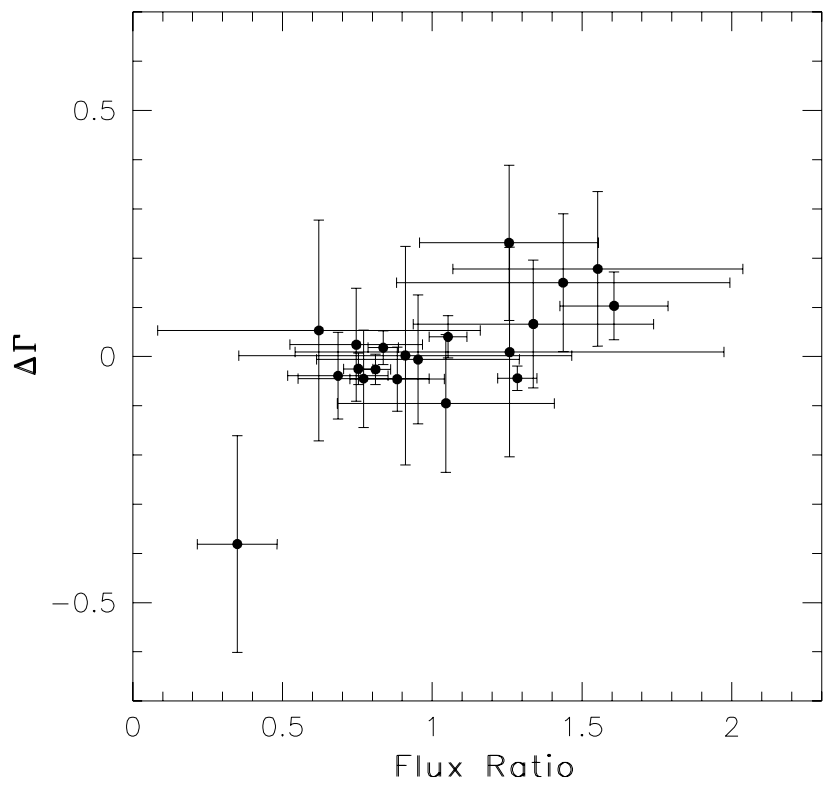

Fig. 4. Photon index difference between 8.5 -yr and 3FGL values vs. flux ratio $\left(\right.$ Flux $_{8.5-y r} /$ Flux $_{3 \text { FGL }}$ ) is shown.

already observed for the whole sample of blazars reported in the 3LAC (Ackermann et al. 2015), and expected by the blazar sequence proposed by Fossati et al. (1998) and Ghisellini et al. (1998).

Only three HSP sources of our sample (SWIFT J1136+6738, SWIFT J1221+3012, and SWIFT J1428+4234) with very high synchrotron peak frequency (i.e. $10^{17}-10^{18} \mathrm{~Hz}$ ) are included in the 105-month Swift-BAT catalogue (Oh et al. 2018). These three sources have an X-ray photon index in the 14-195 $\mathrm{keV}$ energy range of 2.3-2.9 and a corresponding $\gamma$-ray photon index of 1.5-1.7, indicating that we are observing the declining part of the synchrotron emission in hard X-rays and the rising part of the inverse Compton emission in $\gamma$ rays.

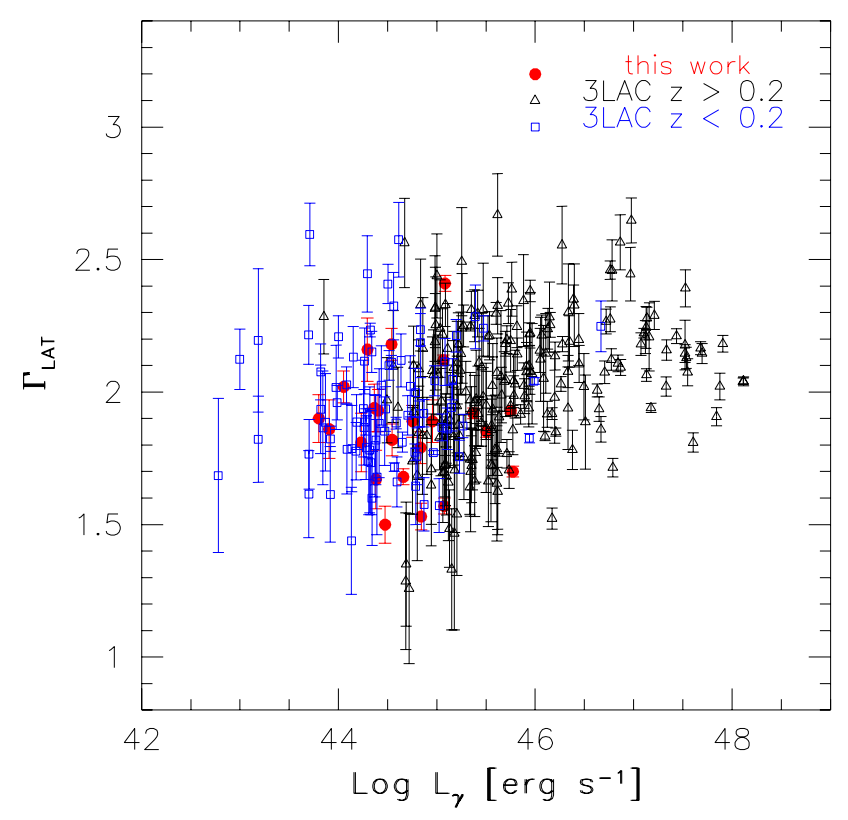

Fig. 5. Photon index vs. $\gamma$-ray luminosity in the $0.1-300 \mathrm{GeV}$ energy range. Red stars: BL Lacs detected from our sample, black open triangles: BL Lacs in the 3LAC with $z>0.2$, blue open squares: BL Lacs in the $3 \mathrm{LAC}$ with $z<0.2$.

\subsection{Comparison with $3 F H L$}

In addition to the LAT catalogues with low-energy threshold of $0.1 \mathrm{GeV}$, three hard-source catalogues have been released, the most recent one being the 3FHL, based on $7 \mathrm{yr}$ of data analysed in the $10 \mathrm{GeV}-2 \mathrm{TeV}$ energy range. The 3FHL contains 1556 objects and takes advantage of the improvement provided by Pass 8 data by using the PSF-type event classification. The vast majority of detected sources (79\%) are associated with extragalactic counterparts at lower energies; in particular, BL Lacs are the dominant extragalactic population (i.e. 750 objects). Among the $23 \mathrm{BL}$ Lacs detected by Fermi-LAT 


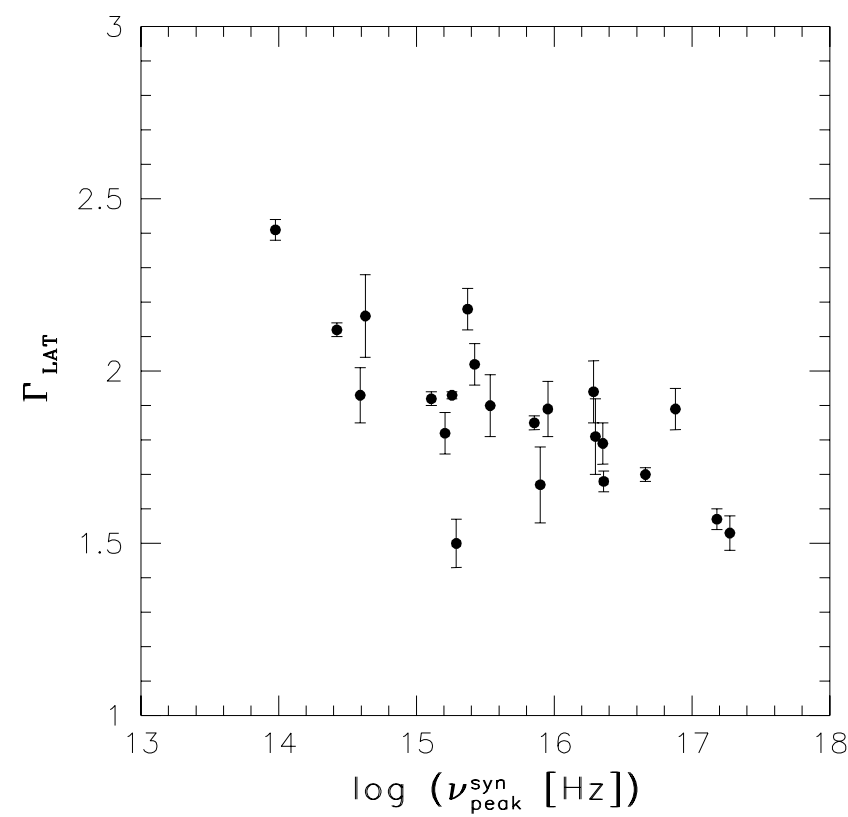

Fig. 6. $\gamma$-ray photon index vs. synchrotron peak frequency of the 22 LAT-detected sources in our sample with $v_{\text {peak }}$ estimated.

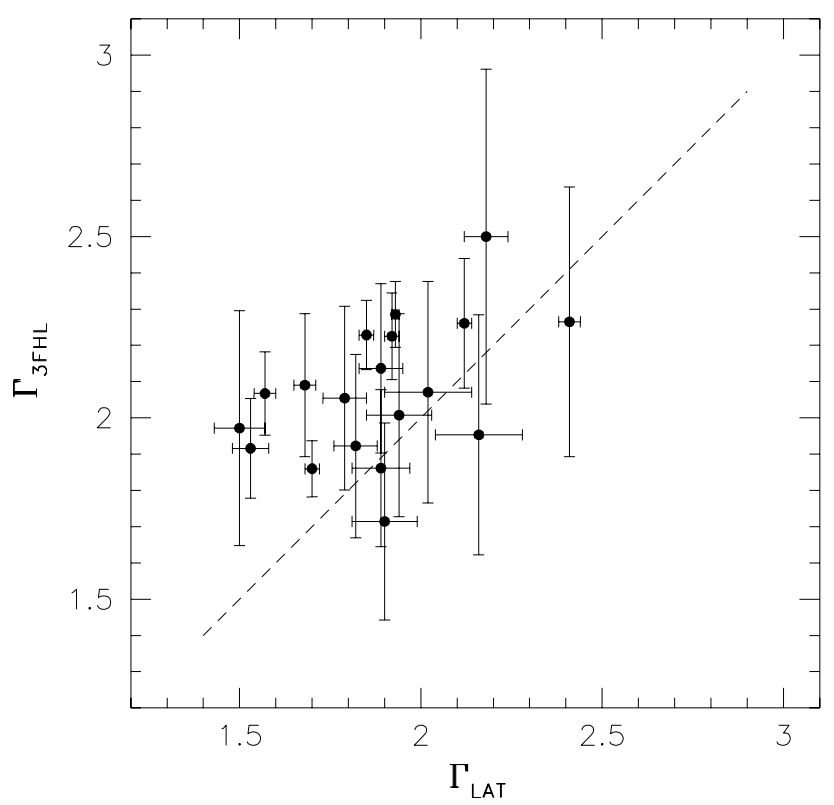

Fig. 7. 3FHL photon index vs. photon index estimated over $8.5 \mathrm{yr}$ in the $0.1-300 \mathrm{GeV}$ energy range. A one-to-one reference short dashed line is plotted.

in the $0.1-300 \mathrm{GeV}$ energy range, 19 are included in the 3FHL. This is not surprising, considering that most of the LAT-detected sources in our sample are HSP, with the high-energy peak within the energy range covered by the 3FHL. All sources of our sample included in the 3FHL are also detected in the $0.1-300 \mathrm{GeV}$ energy range. Among the 19 sources in the 3FHL, 9 are also detected at very high energy (VHE; $E>100 \mathrm{GeV}$ ) by the current Imaging Atmospheric Cherenkov Telescopes ${ }^{5}$. Moreover, 11 out 19 3FHL sources are included also in the 2FHL (Ackermann et al. 2016), where a higher energy threshold of $50 \mathrm{GeV}$ was considered for the analysis. Four 2FHL sources in

\footnotetext{
TeVCat v3.400: http://tevcat. uchicago.edu/
}

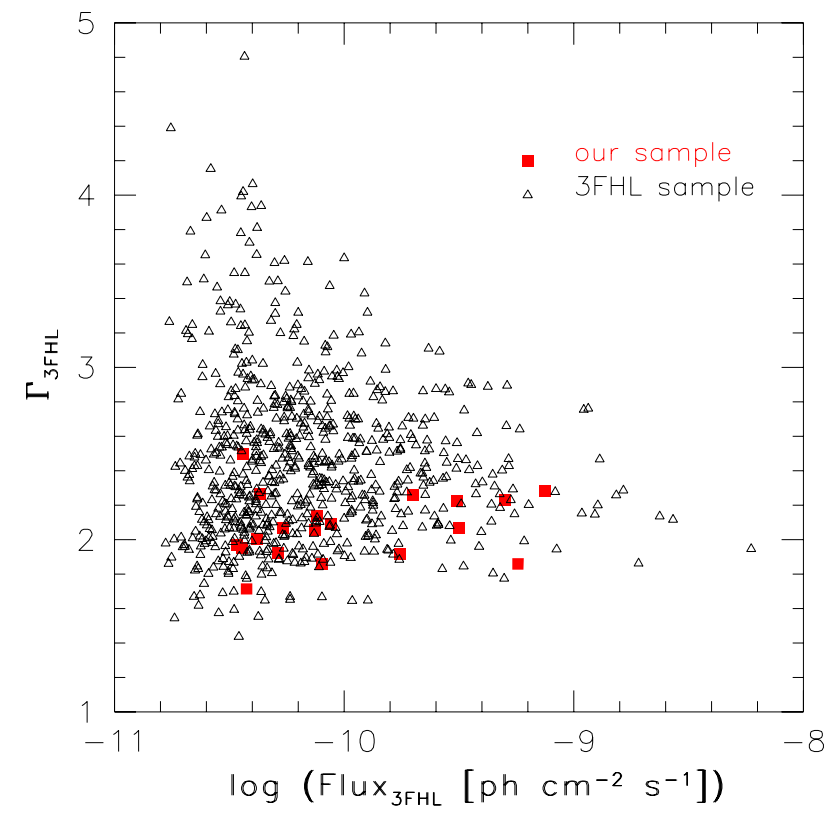

Fig. 8. Photon index vs. flux of the BL Lacs included in the 3FHL. Red squares show the BL Lacs included in our sample.

our sample (2FHL J0809.5+3455, 2FHL J1053.5+4930, 2FHL $\mathrm{J} 1058.5+5625$, 2FHL J1120.8+4212) are not detected at VHE and could be good targets for the current Cherenkov Telescopes.

The difference between the 3FHL photon index and the photon index estimated in this work in the $0.1-300 \mathrm{GeV}$ energy range ranges between -0.21 and 0.50 . In Fig. 7 we compare the 3FHL photon index with the LAT photon index in the $0.1-300$ $\mathrm{GeV}$ energy range. The average 3FHL photon index of the 19 sources in our sample is $\left\langle\Gamma_{3 \mathrm{FHL}}\right\rangle=2.07 \pm 0.23$. As a comparison the average photon index estimated in this work in the 0.1$300 \mathrm{GeV}$ energy range is $\left\langle\Gamma_{8.5-\mathrm{yr}}\right\rangle=1.88 \pm 0.05$, indicating a small difference between the photon index estimated in the two energy ranges. The difference between the two photon indices of the 19 LAT-detected sources in both samples is $-0.21<$ $\Delta \Gamma_{3 \mathrm{FHL}} \equiv\left(\Gamma_{3 \mathrm{FHL}}-\Gamma_{8.5 \mathrm{yr}}\right)<0.50$, suggesting that the average $\gamma$-ray spectrum of these sources below $300 \mathrm{GeV}$ connects rather smoothly with the spectrum in the $10 \mathrm{GeV}-2 \mathrm{TeV}$ energy range. For five sources, even considering the uncertainties, the 3FHL photon index is larger than the 3FGL photon index, suggesting a possible curvature in the overall $\gamma$-ray spectrum. Removing these five sources, the average photon index estimated in the $0.1-300 \mathrm{GeV}$ energy range $\left(\left\langle\Gamma_{8.5-\mathrm{yr}}\right\rangle=1.99 \pm 0.06\right)$ and in the $10 \mathrm{GeV}-2 \mathrm{TeV}$ energy range $\left(\left\langle\Gamma_{3 \mathrm{FHL}}\right\rangle=2.11 \pm 0.25\right)$ are still compatible. In case of sources with $\Delta \Gamma_{3 \mathrm{FHL}}<0$, and therefore a harder 3FHL photon index value with respect to the $0.1-$ $300 \mathrm{GeV}$ value, the two photon indexes are compatible within the uncertainties, as shown in Fig. 7. This is an indication of no significant break or softening in the observed spectra between $0.1 \mathrm{GeV}$ and $2 \mathrm{TeV}$. This should be related also to the small effect of absorption due to interactions with the extragalactic background light (EBL, Dominguez et al. 2011) on the spectra of BL Lacs at low redshift, such as those in our sample (see e.g. Dominguez \& Ajello 2015).

In Fig. 8, we plot the $\gamma$-ray photon index versus flux in the $10 \mathrm{GeV}-2 \mathrm{TeV}$ energy range of the BL Lacs included in the 3FHL, with the $19 \mathrm{BL}$ Lacs of our sample shown with filled red squares. The sources in our sample detected above $10 \mathrm{GeV}$ have a photon index $\Gamma_{3 \mathrm{FHL}}<2.6$, independently from the flux. 


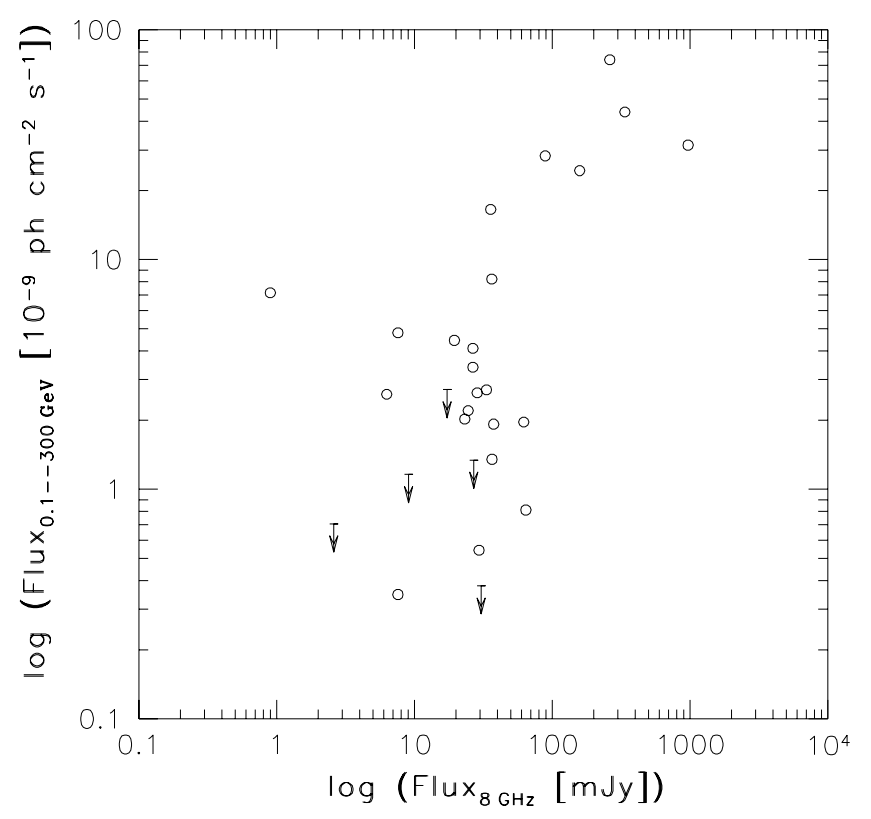

Fig. 9. Flux density at $8 \mathrm{GHz}$ vs. $\gamma$-ray flux estimated in the $0.1-$ $300 \mathrm{GeV}$ energy range over $8.5 \mathrm{yr}$ of LAT data. Arrows show 2- $\sigma$ upper limits of the $\gamma$-ray flux.

Therefore, the photon index seems to be more important than the flux for detecting sources at high energies, at least when considering a sample in the same bin of redshift, that is, with similar effect of absorption due to interactions with the EBL. All the 3FHL sources in our sample are good candidates for future observations with the Cherenkov Telescope Array (Acharya et al. 2018).

\subsection{Comparison with VLBI data at 8 and $15 \mathrm{GHz}$}

Radio and $\gamma$-ray emission are strongly correlated in blazars (e.g. Ghirlanda et al. 2010; Ackermann et al. 2011; Richards et al. 2014; Mufakharov et al. 2015; Böck et al. 2016). However, given the small field of view of VLBI arrays, large surveys with milliarcsecond angular resolution are not available and for parsec scale studies one has to resort to dedicated pointed observations. Linford et al. (2012) present VLBI data for a large sample of $\gamma$-ray sources, including as many as $95 \mathrm{BL}$ Lacs; interestingly, they find that LAT-detected BL Lacs do not differ significantly from a control sample of non-LAT-detected BL Lacs in terms of brightness and morphology.

The results of dual frequency ( 8 and $15 \mathrm{GHz}$ ) VLBA observations for the sources in our sample were presented in Paper I. Compact components were found for 27 sources. The presence of $\gamma$-ray emission and of a compact radio component seem to be well connected: 21 of the 23 LAT-detected sources $(91 \%)$ reported in this paper have a VLBI component with respect to 6 of the 19 non-LAT-detected sources (32\%). The only LATdetected sources undetected at parsec scale are J0847+1133 and $\mathrm{J} 1534+3715$. However, the lack of detection of $\mathrm{J} 0847+1133$ is likely due to observational problems during VLBI observations reported in Paper I. Nevertheless, a compact emission has been observed with the European VLBI Network (EVN) at $5 \mathrm{GHz}$ (Mantovani et al. 2015) and with VLBA at $8 \mathrm{GHz}$ (Bourda et al. 2010) for this source. On the other hand, no radio emission has been revealed at both $8 \mathrm{GHz}$ and $15 \mathrm{GHz}$ for J1534+3715 with VLBA. It is worth noticing that this source has the lowest NVSS flux densities at $1.4 \mathrm{GHz}$ among the

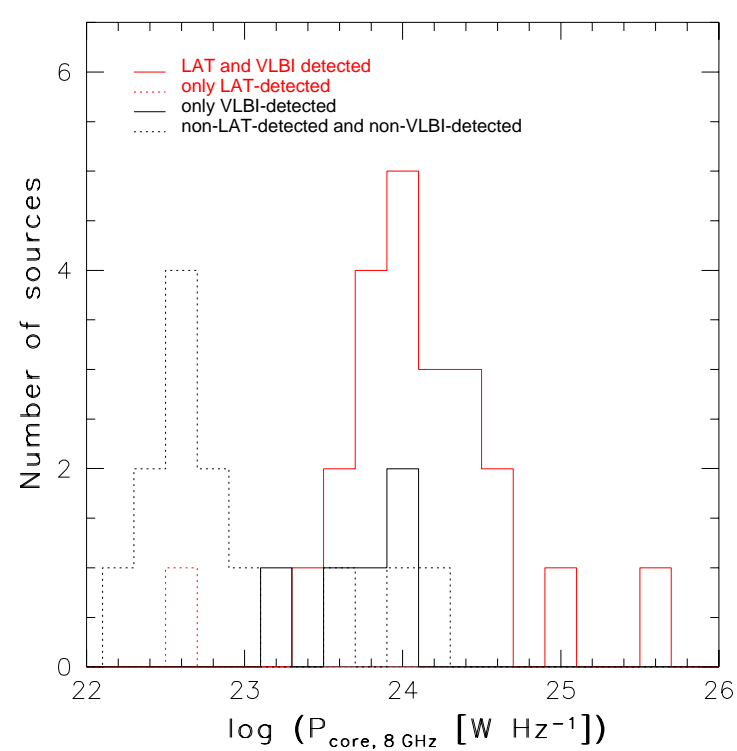

Fig. 10. Histogram of the core power at $8 \mathrm{GHz}$ of the sources in our sample. Solid line indicates that the source is detected and short-dash line shows the upper limit. Red lines refer to LAT-detection and black lines refer to VLBI-detection.

LAT-detected sources in our sample, suggesting that the radio emission is not concentrated in the core region but extended emission is present on scales which are not probed by VLBI arrays.

The six non-LAT-detected sources with a compact component detected by VLBA have a $8 \mathrm{GHz}$ flux density in the range $2.6-30.0 \mathrm{mJy}$ with respect to the range of flux densities of 6.0 $969.5 \mathrm{mJy}$ for the LAT-detected sources. This result suggests that these sources are in the low-values part of the distribution of $8 \mathrm{GHz}$ flux density in our sample. Comparing the $\gamma$ ray flux in the $0.1-300 \mathrm{GeV}$ energy range with the $8 \mathrm{GHz}$ flux density of the 27 sources for which parsec scale emission is detected by VLBA (Fig. 9), a connection between the radio and $\gamma$-ray emission is evident, in agreement with the radio $/ \gamma$-ray flux correlation reported in Ackermann et al. (2011). We calculate a Pearson correlation coefficient between $8 \mathrm{GHz}$ flux densities and $\gamma$-ray fluxes and obtain $R=0.5746$, indicating a moderate positive correlation $(P$-value $=0.001384)$.

In Fig. 10 we compare the VLBA core power at 8 $\mathrm{GHz}$ for 41 sources $(\mathrm{J} 0751+2913$ has not been observed at $8 \mathrm{GHz}$ ). Upper limits are reported for 14 sources (see Paper I, Table 5 for details); all of them are non-LAT-detected sources, except for J1534+371. The LAT-detected sources have on average a higher core power at $8 \mathrm{GHz},\left\langle P_{\text {core, } 8 \mathrm{GHz}}^{\mathrm{LAT}}\right\rangle=$ $1.3 \times 10^{24} \mathrm{~W} \mathrm{~Hz}^{-1}$, with respect to non-LAT-detected sources, $\left\langle P_{\text {core, } 8 \mathrm{GHz}}^{\text {non-LAT }}\right\rangle=1.4 \times 10^{23} \mathrm{~W} \mathrm{~Hz}^{-1}$. All sources with $P_{\text {core, } 8 \mathrm{GHz}}$ $>2 \times 10^{24} \mathrm{~W} \mathrm{~Hz}^{-1}$ have been detected in $\gamma$ rays by LAT. On the other hand, only one source with $P_{\text {core, } 8 \mathrm{GHz}}<3 \times$ $10^{23} \mathrm{~W} \mathrm{~Hz}^{-1}$, J1534+3715, has been detected by LAT. This indicates that LAT-detected sources are the most luminous radio sources of our sample on parsec scale. Moreover, it suggests that there is a population of BL Lacs that lack a VLBI core, have a low $\gamma$-ray emission, and likely have a small Doppler factor. This is even more evident by comparing the $\gamma$-ray luminosity in the $0.1-300 \mathrm{GeV}$ energy range with the radio luminosity of the core at $8 \mathrm{GHz}$ (Fig. 11): the bottom-left part of the plot is populated by sources with a low luminosity in both the radio and $\gamma$-ray bands. All the sources that lie in that part of the 


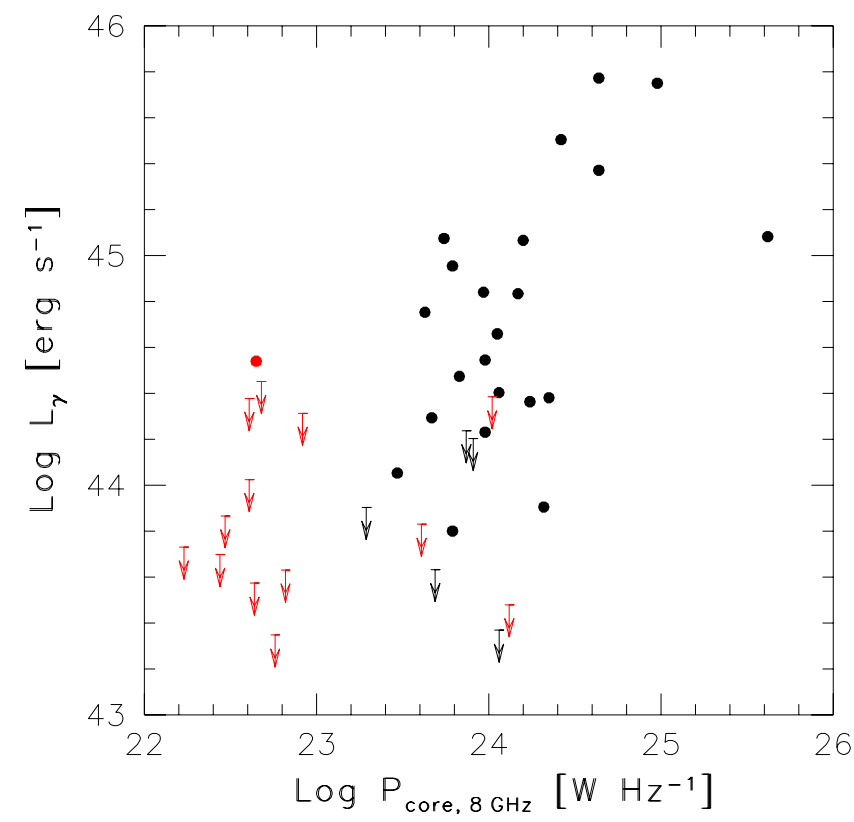

Fig. 11. Luminosity of the core at $8 \mathrm{GHz}$ vs. $\gamma$-ray luminosity in the $0.1-300 \mathrm{GeV}$ energy range for the sources in our sample. Filled circles indicate LAT-detected sources, while arrows show upper limits of the $\gamma$ ray luminosity. If only an upper limit on the radio luminosity has been estimated, the data are reported in red.

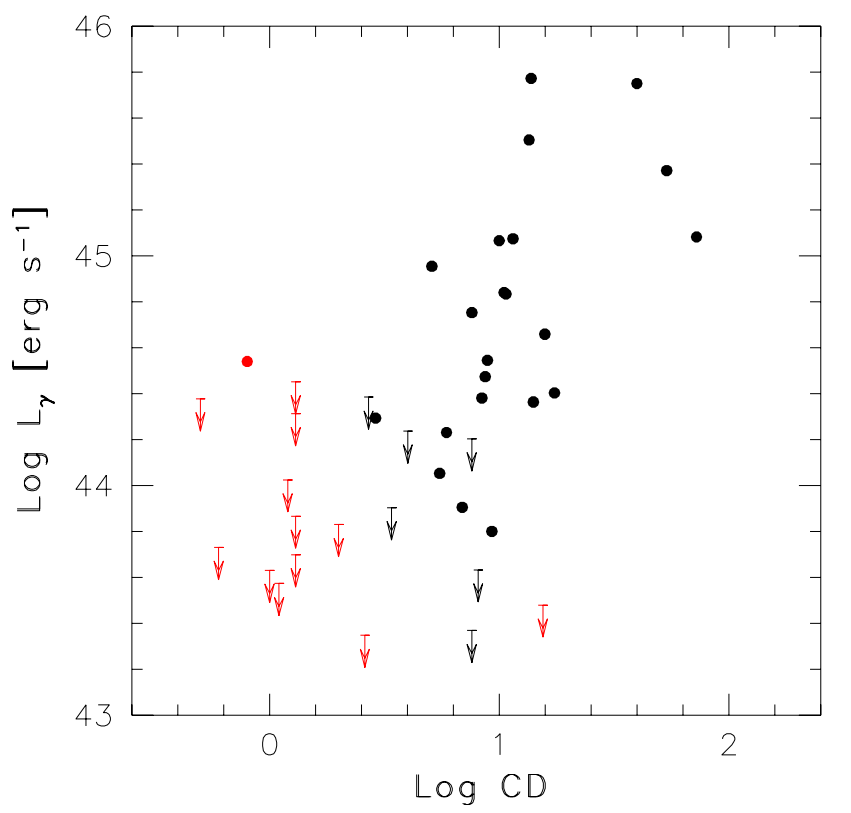

Fig. 12. Core dominance vs. $\gamma$-ray luminosity in the $0.1-300 \mathrm{GeV}$ energy range for the sources in our sample. Filled circles indicate LATdetected sources, while arrows show upper limits of the $\gamma$-ray luminosity. If only an upper limit on the CD has been estimated, the data are reported in red.

luminosity-luminosity plot are not yet detected by Fermi-LAT, except for $\mathrm{J} 1534+3715$.

On the basis of the radio properties (i.e. source compactness, Core Dominance, $\mathrm{CD}^{6}$, source morphology and radio spectrum) in Paper I, all the sources have been classified in three groups: Doppler-dominated (DD, 23 cases), lobe-dominated (LD, 11

\footnotetext{
${ }^{6} \mathrm{CD}$ is defined as ratio between the observed core radio power and the
} estimated unbeamed total radio power at low frequency. cases), and undetermined type (U, 8 cases) sources. In DD sources, the luminosity of the core is much larger than the luminosity of the extended component, implying relativistic jets and small viewing angles; LD sources have less prominent or undetected cores, and significant extended emission; U sources are undetected with VLBI but their overall flux density is so low that it does not provide stringent limits on the ratio between nuclear and extended emission. Among the 23 LAT-detected sources, 20 are classified as DD, and 3 as LD (J0847+1133, J1341+3959, and $\mathrm{J} 1534+3715)$. All three newly detected $\gamma$-ray sources are classified as DD. European VLBI Network and VLBA observations at $5 \mathrm{GHz}$ and $8 \mathrm{GHz}$, respectively, and a CD of 5.1 suggests an intermediate classification of J0847+1133 between LD and DD. Faint radio emission has been detected with VLBA observations of the other two LAT-detected sources classified as LD. On the other hand, three sources classified as DD for their radio properties (J0754+3910, J0903+4055, J1516+2918) are not detected in $\gamma$ rays by LAT up to now. These three sources have a relatively low CD (7.6-8.0) with respect to the LATdetected sources classified as DD (5.5-72.4). Considering that $\mathrm{CD}$ is a strong indication of Doppler beaming of the emission, this suggests that the DD sources not detected by LAT have a lower Doppler factor with respect to the LAT-detected ones. In Fig. 12, the CD versus $\gamma$-ray luminosity of sources in our sample has been shown. The plot confirms that sources with large $\gamma$-ray luminosity also have high $\mathrm{CD}$ values. In the cases of $\mathrm{J} 0754+3910$ and J0903+4055, the upper limits on the $\gamma$-ray flux estimated over $8.5 \mathrm{yr}$ of LAT observations are compatible with the sensitivity limit estimated over $10 \mathrm{yr}$ of LAT observations, indicating that, accumulating more data, these sources may be detected by the LAT in the near future.

\section{Summary}

With the advent of the Fermi satellite, our understanding of the radio and $\gamma$-ray connection in blazars significantly increased. BL Lacs are found to be the most abundant class of extragalactic objects observed by LAT. However, they are relatively weak radio sources and the parsec-scale structure has not been studied in detail for most of them. We have selected a sample of 42 BL Lacs included in the BZCAT catalogue with $z<0.2$, located within the sky area covered by SDSS, and with no selection limit on $\gamma$-ray flux. Most of the sources (31/42) have a $1.4 \mathrm{GHz}$ flux density that is lower than the sensitivity limit of VIPS (i.e. 85 mJy). These sources have been observed with the VLBA at 8 $\mathrm{GHz}$ and $15 \mathrm{GHz}$, and the results are reported in Liuzzo et al. (2013). In this paper we have investigated the $\gamma$-ray properties of the radio fainter BL Lac sources and the connection with the radio properties.

Among the sources in the sample, 23 out of 42 have been detected by our LAT analysis, with an average photon index $\langle\Gamma\rangle=1.87 \pm 0.06$ and a luminosity varying between $6 \times 10^{43}$ and $6 \times 10^{45} \mathrm{erg} \mathrm{s}^{-1}$, a range of values typical of $\gamma$-ray emitting BL Lacs and within the range of values observed for the other BL Lacs with $z<0.2$ in the 3LAC. All sources reported in the 3FGL are confirmed by our analysis. In addition we have revealed three new sources with respect to the 3FGL. These three new detections have fluxes below $1.4 \times 10^{-8} \mathrm{ph} \mathrm{cm}^{-2} \mathrm{~s}^{-1}$ and photon index 1.7-1.9.

Based on the $v_{\text {peak }}^{\text {syn }}, 30$ sources in our sample are classified as HSP; in particular 19 out of 23 LAT-detected sources are HSP BL Lacs. An anti-correlation between $v_{\text {peak }}^{\text {syn }}$ and $\gamma$-ray photon index is observed for the LAT-detected BL Lacs, in agreement with the blazar sequence. Among the 23 
LAT-detected sources, 19 are included in the 3FHL, for which a small difference between the photon index estimated in the $0.1-$ $300 \mathrm{GeV}$ and $10 \mathrm{GeV}-2 \mathrm{TeV}$ energy range has been observed. This suggests that the average $\gamma$-ray spectrum of these sources connects rather smoothly from $0.1 \mathrm{GeV}$ to $2 \mathrm{TeV}$, as expected due to the low EBL attenuation at $z<0.2$.

A compact radio component in the VLBA images has been detected in 22 out of the 23 LAT-detected sources, and only 6 out of 19 non-LAT-detected sources. LAT-detected BL Lacs are more luminous on parsec scales with respect to non-LATdetected sources and tend to have larger core dominance according to the Unified models. All sources with $P_{\text {core } 8 \mathrm{GHz}}>2 \times$ $10^{24} \mathrm{~W} \mathrm{~Hz}{ }^{-1}$ have been detected in $\gamma$ rays by LAT, confirming the strong correlation between radio and $\gamma$-ray properties. In the same way, we have identified a possible population of lowluminosity BL Lacs in both the radio and $\gamma$ rays, lacking a VLBI core, and with a small Doppler factor. This highlights an opportunity for future $\gamma$-ray observations to discover low-luminosity BL Lacs with Fermi-LAT and the next generation of $\gamma$-ray satellites.

Radio and $\gamma$-ray emission in blazars are both related to the presence of relativistic particles in jets. Among the 23 LATdetected sources, 20 are classified as DD, indicating the presence of a relativistic jet in radio images. All these characteristics confirm that LAT-detected BL Lacs are dominated by Doppler boosting effects. However, three LAT-detected sources are classified as LD, with no evidence of relativistic jet with high Doppler factor in radio images and relatively low core dominance, showing non-classical properties for a $\gamma$-ray-emitting BL Lac. The properties of these peculiar sources will be investigated in detail by means of multi-frequency observations.

Acknowledgements. We acknowledge financial contribution from gran PRIN-INAF-2011. This work was supported by the National Research Council of Science \& Technology (NST) granted by the International joint research project (EU-16-001). We thank the anonymous referee, M. Kadler, M. Orienti, A. Dominguez, R. Caputo, and D. Thompson for useful comments and suggestions. The Fermi-LAT Collaboration acknowledges generous ongoing support from a number of agencies and institutes that have supported both the development and the operation of the LAT as well as scientific data analysis. These include the National Aeronautics and Space Administration and the Department of Energy in the United States, the Commissariat à l'Energie Atomique and the Centre National de la Recherche Scientifique/Institut National de Physique Nucléaire et de Physique des Particules in France, the Agenzia Spaziale Italiana and the Istituto Nazionale di Fisica Nucleare in Italy, the Ministry of Education, Culture, Sports, Science and Technology (MEXT), High Energy
Accelerator Research Organization (KEK) and Japan Aerospace Exploration Agency (JAXA) in Japan, and the K. A. Wallenberg Foundation, the Swedish Research Council and the Swedish National Space Board in Sweden. Additional support for science analysis during the operations phase is gratefully acknowledged from the Istituto Nazionale di Astrofisica in Italy and the Centre National d'Études Spatiales in France. This work performed in part under DOE Contract DE-AC02-76SF00515.

\section{References}

Abazajian, K. N., Adelman-McCarthy, J. K., Agueros, M. A., et al. 2009, ApJS, 182,543

Abdo, A. A., Ackermann, M., Ajello, M., et al. 2010, ApJ, 715, 429

Acero, F., Ackermann, M., Ajello, M., et al. 2015, ApJS, 218, 23

Acero, F., Ackermann, M., Ajello, M., et al. 2016, ApJS, 223, 2

Acharya, B.S., Agudo, I., Al Samarai, I., et al. 2018, ApJ, submitted

Ackermann, M., Ajello, M., Allafort, A., et al. 2011, ApJ, 741, 30

Ackermann, M., Ajello, M., Atwood, W., et al. 2015, ApJ, 810, 14

Ackermann, M., Ajello, M., Atwood, B., et al. 2016, ApJS, 222, 5

Ade, P. A. R., Aghanim, N., Arnaud, M., et al. 2016, A\&A, 594, A63

Ajello, M., Atwood, W. B., Baldini, L., et al. 2017, ApJS, 232, 18

Arsioli, B., \& Chang, Y.-L. 2017, A\&A, 598, A134

Atwood, W. B., Abdo, A. A., Ackermann, M., et al. 2009, ApJ, 697, 1071

Atwood, W. B., Albert, A., Baldini, L., et al. 2013, Instrum. Methods Astrophys., submitted

Böck, M., Kadler, M., Muller, C., et al. 2016, A\&A, 590, A40

Bourda, G., Charlot, P., Porcas, R. W., \& Garrington, S. T. 2010, A\&A, 520, A113

Chang, Y.-L., Arsioli, B., Giommi, P., \& Padovani, P. 2017, A\&A, 598, A17

Dominguez, A., \& Ajello, M. 2015, ApJ, 813, L34

Dominguez, A., Primack, J. R., Rosario, D. J., et al. 2011, MNRAS, 410, 2556

Fossati, G., Maraschi, L., Celotti, A., Comastri, A., \& Ghisellini, G. 1998 MNRAS, 299, 433

Ghirlanda, G., Ghisellini, G., Tavecchio, F., \& Foschini, L. 2010, MNRAS, 407, 791

Ghisellini, G., Celotti, A., Fossati, G., Maraschi, L., \& Comastri, A. 1998, MNRAS, 301, 451

Ghisellini, G., Righi, C., Costamante, L., \& Tavecchio, F. 2017, MNRAS, 469, 255

Helmboldt, J. F., Taylor, G. B., Tremblay, S., et al. 2007, ApJ, 658, 203

Jorstad, S., Marscher, A. P., Morozova, D. A., et al. 2017, ApJ, 846, 98

Linford, J. D., Taylor, G. B., Romani, R. W., et al. 2012, ApJ, 744, 177

Liuzzo, E., Giroletti, M., Giovannini, G., et al. 2013, A\&A, 560, A23

Mantovani, F., Bondi, M., Mack, K.-H., et al. 2015, A\&A, 577, A36

Massaro, E., Giommi, P., Leto, C., et al. 2009, A\&A, 495, 691

Mattox, J. L., Bertsch, D. L., Chiang, J., et al. 1996, ApJ, 461, 691

Mufakharov, T., Mingaliev, M., Sotnikova, Yu., Naiden, Ya., \& Erkenov, A. 2015 MNRAS, 450, 2658

Oh, K., Koss, M., Markwardt, C. B., et al. 2018, ApJS, 235, 4

Press, W. H., Flannery, B. P., Teukolsky, S. A., \& Vetterling, W. T. 1992, Numerical Recipes in FORTRAN 77, 2nd edn. (Cambridge: Cambridge University Press)

Richards, J. L., Hovatta, T., Max-Moerbeck, W., et al. 2014, MNRAS, 438, 3058 